\title{
CLIMAT, MICROCLIMAT ET PRODUCTION LIGNEUSE
}

\author{
G. AUSSENAC \\ Station de Sylviculture et de Production, \\ Centre national de Recherches forestières, I. N. R. A., \\ Champenoux 54370 Einville
}

\section{RÉSUMÉ}

L'action combinée des paramètres climatiques et plus particulièrement des facteurs thermiques et hydrométriques règle les conditions d'existence des arbres et détermine d'ailleurs la distribution géographique des essences et des types de forêts.

Pour la foresterie les recherches entreprises dans ce domaine doivent aboutir à une plus grande maîtrise de la forêt en précisant : les potentialités forestières des différents milieux, les exigences climatiques des principales essences et les moyens d'améliorer les relations arbre-microclimat.

Les facteurs climatiques ou microclimatiques ont deux modes d'action :

- une action accidentelle (accidents climatiques),

- une action permanente (action physiologique).

Les relations entre le climat et la production ligneuse ont été étudiées à partir des indices de production, tel l'indice CVP. de PATERson qui est un outil intéressant pour l'économiste ou le planificateur soucieux d'évaluer approximativement les potentialités de production d'un pays. En fait, la prévision de la production ligneuse potentielle est difficile à faire car d'une part les forêts sont la plupart du temps installées sur les sols non utilisés par l'agriculture (en général les moins fertiles) et d'autre part l'introduction d'essences exotiques peut relever le niveau de cette production.

A l'échelle régionale les études de potentialités forestières peuvent apporter des informations intéressantes mais elles sont limitées par le manque de données climatologiques. Aussi dans ce domaine la progression des connaissances sera difficile.

Au niveau du massif forestier ou de la parcelle le couvert influence les facteurs climatiques et l'on parle de microclimat. Les études microclimat-production doivent de ce fait tenir compte de ces influences. Des notions particulières interviennent : la structure des houppiers et des peuplements, l'environnement de la parcelle étudiée.

De nombreuses études ont été réalisées en conditions contrôlées et ont permis de préciser les relations des processus photosynthétiques avec les facteurs climatiques. Mais il faut aussi nécessairement travailler en forêt. En effet d'une part l'action du couvert forestier influence le climat et d'autre part dans la nature les facteurs agissent de façon conjuguée et non séparée. La compréhension de ces phénomènes passe par deux types de recherches qui sont menées la plupart du temps simuitanément ainsi que nous le faisons à Nancy :

- l'analyse des influences de la structure des peuplements sur les facteurs du microclimat, - l'étude de la réponse des arbres à ces facteurs, appréciée par la croissance, la production ou
la photosynthèse. 
De même l'étude du fonctionnement du couver forestier permet d'envisager des applications possibles : rôle de l'élagage artificiel des arbres forestiers, amélioration du potentiel d'assimilation nette par utilisation d'essence à morphologie des houppiers plus efficace.

\section{1. - INTRODUCTION}

Il est hors de doute que le climat a une action importante et décisive sur la croissance de la forêt. Aucun forestier d'ailleurs ne contestera ce fait. L'action combinée des paramètres climatiques, et plus particulièrement des facteurs thermiques et hydrométriques, règle les conditions d'existence des arbres et détermine la distribution géographique des essences et des types de forêts. Lorsqu'on parle de relations climat-production ligneuse, un problème d'échelle apparaît. Si l'on s'intéresse aux formations forestières du monde ou d'un pays les facteurs climatiques pourront être considérés comme déterminants; par contre, si l'on considère un massif forestier ou une parcelle, les interactions climat végétation déterminent différents microclimats forestiers, qui conditionnent en partie le développement et la croissance des arbres.

Pour la foresterie, les recherches entreprises dans ce domaine doivent aboutir à une plus grande maîtrise de la forêt en précisant : les potentialités forestières des différents milieux, les exigences climatiques des principales essences et les moyens d'améliorer les relations arbre-microclimat.

Les facteurs climatiques ou microclimatiques peuvent avoir schématiquement deux modes d'action :

— une action : qui peut être due à la température, au vent, à la neige, ou aux brouillards givrants;

- une action permanente : qui est le fait de l'eau, du rayonnement, de la température, cu vent, de l'état hygrométrique de l'air.

Le premier mode d'action correspond à des accidents climatiques qui peuvent provoquer la mort d'une partie (bourgeons, rameaux, branches, fleurs) ou de la totalité de l'arbre. Ce type d'action peut être très important pour la limitation de la production en montagne ou à la périphérie de l'aire des essences. L'introduction dans certaines régions d'espèces mal adaptées peut avoir pour conséquence une diminution notable de la production : dégâts de neige, chablis. Van MiEgroET (1967) cite à cet égard des chiffres éloquents pour certaines plantations d'épicéa en Belgique. En Grande-Bretagne la production peut être sérieusement limitée par le vent.

L'action qualifiée de permanente est physiologique. Elle correspond à l'action permanente des facteurs climatiques ou microclimatiques sur les processus photosynthétiques. 
De ce fait, leurs conséquences sont plus difficiles à appréhender. Les accidents climatiques peuvent d'ailleurs avoir des prolongements physiologiques difficiles à cerner. Ainsi, une gelée tardive a pour action directe, la destruction des bourgeons d'un jeune sapin pectiné, mais elle a une action indirecte en perturbant la physiologie de l'arbre (perte momentanée de la dominance apicale, ralentissement des phénomènes photosynthétiques par diminution de l'appareil foliaire et de son efficacité) pendant un nombre d'années plus ou moins grand. On sait en effet que la photosynthèse diminue avec l'âge des aiguilles ainsi que l'a montré Freeland (fig. 1).

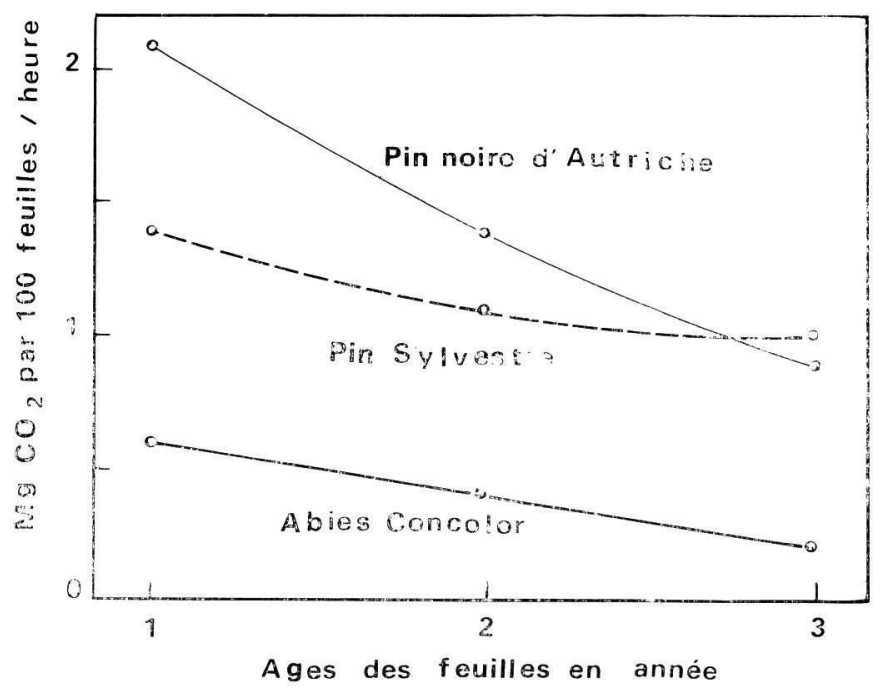

Fig. 1. - Effet de l'àge sur l'assimilation nette des aiguilles (d'après FreELAND 1952)

\section{3. -- RELATIONS ENTRE LE CLIMAT ET LA PRODUCTION LIGNEUSE}

Nous distinguerons les études entreprises sur les relations climat et production, des études microclimat et production. L'observation des forestiers et des botanistes ayant établi une relation entre les facteurs du climat et la végétation forestière, certains chercheurs ont essayé de quantifier ces relations afin de pouvoir prédire la production à partir àun certain nombre de paramètres climatiques qui apparaissent comme les plus importants. Ces travaux ont eu pour cadre, soit le monde entier, soit des pays ou des régions d'étendue plus limitée.

\subsection{Les indices de production}

Pour ce qui est de la prévision de la production à l'échelle d'un pays ou du monde, le Suédois Paterson (1956) a proposé l'indice C.V.P. (climat, Végétation, Production). Cet indice dérive des indices établis par les phytogéographes pour caractériser les climats. 
Mais PATERSON met en corrélation directe le climat et la production ligneuse $\left(\mathrm{m}^{3} / \mathrm{ha} / \mathrm{an}\right)$ de l'origine du peuplement à 100 ans. Il se place dans le cas de forêts naturelles qui se seraient développées sans problème. Il exclut donc ici les limitations et les gains de production qui pourraient intervenir pour des essences introduites. Il définit chaque fois une productivité idéale qui résulterait d'une gestion forestière très évoluée. L'indice C.V.P. est le suivant :

$$
\text { C.V.P. }=\frac{\mathrm{T} v}{\mathrm{~T} a} \times \mathrm{P} \frac{\mathrm{G}}{12} \times \frac{\mathrm{E}}{100}
$$

$\mathrm{T} v$ : Température moyenne en ${ }^{\circ} \mathrm{C}$ du mois le plus chaud,

$\mathrm{T} a$ : différence entre les températures moyennes mensuelles du mois le plus chaud et du mois le plus froid,

$P$ : pluviométrie moyenne annuelle en $\mathrm{mm}$,

G : longueur de la saison de végétation en mois, en climats tempérés ou froids, mois pour lesquels la température moyenne est supérieure à $3{ }^{\circ} \mathrm{C}$; en climats chauds Paterson se réfère à l'indice de MARTONNE :

$$
i=\frac{12 \mathrm{P}}{\mathrm{T}+10} \text { avec, }
$$

$\mathrm{P}$ : pluviosité mensuelle en $\mathrm{mm}$

$\mathrm{T}$ : température moyenne du même mois en ${ }^{\circ} \mathrm{C}$

et alors seuls les mois humides $i>20$ sont pris en compte.

$\mathrm{E}=100 \frac{\mathrm{R}_{p}}{\mathbf{R}_{s}} \quad \begin{array}{ll}\mathrm{R}_{p} \text { : radiation cumulée au pôle } \\ \mathbf{R}_{s}: \text { dans la station considérée }\end{array}$

E varie de 0,40 à l'équateur à 0,90 au nord de la Sibérie.

L'indice varie de 0 dans les régions polaires à 20000 dans les régions tropico-équatoriales; les productions correspondantes seraient alors respectivement de 0 et de $15 \mathrm{~m}^{3} /$ ha/an. A partir de cet indice PAterson a pu tracer une carte mondiale des productions forestières.

Cet indice a beaucoup intéressé les forestiers, et certains d'entre eux en particulier WECK et PARDE, ont proposé d'améliorer sa définition. WECK (1957) proposait : d'exprimer la production potentielle non pas en $\mathrm{m}^{3} / \mathrm{ha} / \mathrm{an}$ mais en poids de matière sèche/ha/an; de calculer la saison de végétation en comptant uniquement les mois humides (indice d'aridité > 20) dont la température moyenne ne descend pas en dessous de $20^{\circ} \mathrm{C}$. Parde (1958, 1959, 1964, 1966) a étudié cet indice et a apporté plusieurs améliorations dont certaines ont été adoptées par la suite par PATERSON :

- hors de la région méditerranéenne, il propose de compter pour la saison de végétation les mois dont la température moyenne est $\geqslant 7^{\circ} \mathrm{C}$;

- en zone méditerranéenne, il retient les mois qui simultanément ont une température moyenne supérieure ou égale à $10^{\circ} \mathrm{C}$ et, selon une idée de Gaussen, une pluviosité en $\mathrm{mm}$ égale à 2 fois au moins la température moyenne mensuelle exprimée en ${ }^{\circ} \mathrm{C}$.

PARDE a pu ainsi diviser la France en régions de productions potentielles différentes. 
Ensuite, à l'aide des données fournies par les dispositifs expérimentaux de la Station de Recherche, il a pu calculer la relation liant l'indice C.V.P. amélioré à la production :

$$
\begin{aligned}
& y=-1,43+0,0267 \text { C.V.P. } \\
& \left(\mathrm{m}^{3} / \text { ha/an }\right) \\
& r=0,98 .
\end{aligned}
$$

Par la suite d'ailleurs, il a publié une carte donnant les régions de France où la production ligneuse est supérieure à $10 \mathrm{~m}^{3} / \mathrm{ha} / \mathrm{an}$.

La confrontation des résultats obtenus par PATERSON et PARDE a mis en évidence le rôle important de la température pour la production dans les hautes latitudes, et des précipitations vers l'équateur. PATERSON a d'ailleurs proposé pour améliorer son indice, d'introduire, pour la Suède seulement, la valeur $\sqrt{\mathrm{P}}$. Dans un pays comme la France, la pluie et la température interviennent également. sion :

L'indice C.V.P. et les améliorations qui lui ont été apportés peuvent prêter à discus-

- tout d'abord l'introduction de la pluviosité annuelle peut bien sûr être contestée, car, selon le régime des pluies, l'efficacité de l'eau sera différente;

- la prévision de la production ligneuse potentielle est en fait très difficile à faire, compte tenu des données disponibles. En effet, les forêts (au moins dans les régions qui ont une agriculture développée) sont installées sur des sols superficiels, et, pour un même climat, il est certain que la production ligneuse serait plus importante sur des sols plus profonds.

Par ailleurs, l'introduction d'essences exotiques pourrait également relever cette production. PARde a essayé de répondre à ces objections en choisissant les essences les plus productrices et des parcelles à sol de 70 à $100 \mathrm{~cm}$ de profondeur.

En fin de compte, l'indice de PATERson apparaît comme un outil surtout intéressant pour l'économiste ou le planificateur soucieux d'évaluer approximativement les potentialités de production forestière d'un pays, particulièrement dans ceux où n'existent pas d'informations plus précises.

\section{2. - Les études de potentialités forestières à l'échelle régionale}

Dans les dernières années, des recherches ont été entreprises pour préciser les relations station-production à l'échelle régionale. Ces études font intervenir des données pédologiques et climatiques et elles se sont développées avec la vulgarisation des méthodes statistiques (régression progressive, analyse en composantes principales) et la possibilité de traiter des données nombreuses par ordinateur.

Un exemple intéressant est l'étude d'Oswald (1969) sur les potentialités forestières de la Haute-Ardèche. Il a pu établir une liaison significative (fig. 2) entre : l'altitude et la production totale en $\mathrm{m}^{3}$, l'altitude et la hauteur dominante. La production passe par un maximum vers $1150 \mathrm{~m}$ puis baisse avec l'altitude. L'absence de données climatiques relatives à chaque station considérée n'a pas permis d'étudier les relations directes entre facteurs du climat et production. En Haute-Ardèche, Oswald a montré qu'il existait une relation 
linéaire entre la température et l'altitude; il en est de même pour la durée de la saison de végétation $\left(^{(}\right)$. Finalement, il est difficile de connaître les facteurs responsables des variations de la production avec l'altitude. Il est probable que la température joue un rôle important, de même que la durée de la saison de végétation. Le vent a également un rôle déterminant, sa vitesse augmente avec l'altitude et, à un niveau qui varie avec la morphologie d'ensemble du massif montagneux, il entraîne la disparition de la végétation forestière par « l'effet de sommet ». L'effet du vent sur les peuplements forestiers peut être mécanique ou physiologique. La pluviosité augmente avec l'altitude, en passant généralement par un optimum pluvial. Il est alors possible d'imaginer que l'optimum de production qu'OswaLd a observé soit lié à deux phénomènes, d'une part à une amélioration du bilan hydrique avec l'altitude, qui aurait pour conséquence d'augmenter la production (effet favorable), et, d'autre part, à un effet défavorable de la diminution de température et de l'augmentation de la vitesse du vent qui, au-delà de $1100 \mathrm{~m}$, contrebalancerait l'effet positif du bilan hydrique.

S'il est possible d'évaluer précisément la croissance, la production ligneuse, il est très difficile de disposer de données climatologiques pour les stations étudiées, et ceci est une caractéristique générale des études de ce type. II faut alors utiliser les résultats de postes lointains, ce qui enlève toute précision à l'étude entreprise. Les difficultés seront d'autant plus grandes que l'on se trouvera en régions montagneuse.

On conçoit donc que dans ce domaine la progression des connaissances ne puisse être que difficile.

\section{4. - MICROCLIMAT ET PRODUCTION FORESTIÈRE}

Au niveau du massif forestier ou de la parcelle, le couvert influence les facteurs climatiques et il est alors permis de parler de microclimat. Les études microclimat-production doivent de ce fait tenir compte de ces influences. Des notions particulières interviennent :

- la structure des houppiers et des peuplements,

- l'environnement de la parcelle étudiée.

\section{Étude des relations microclimat-production ligneuse}

\section{Étude dendroclimatologique.}

Des résultats ont pu être obtenus par des méthodes qui relèvent de la dendroclimatologie. Elles consistent à établir des corrélations entre des facteurs climatiques simples ou synthétiques de différentes années et la croissance en longueur ou en diamètre des mêmes années; ce qui impose la nécessité absolue de dater avec précision le cerne ou la pousse annuelle.

Pour la croissance en longueur, le bourgeon terminal de la flèche se forme à la fin de la saison de végétation (août-septembre) chez la plupart des essences de notre pays. Il est

1. Calculée selon les critères retenus par PARDE (1959). 


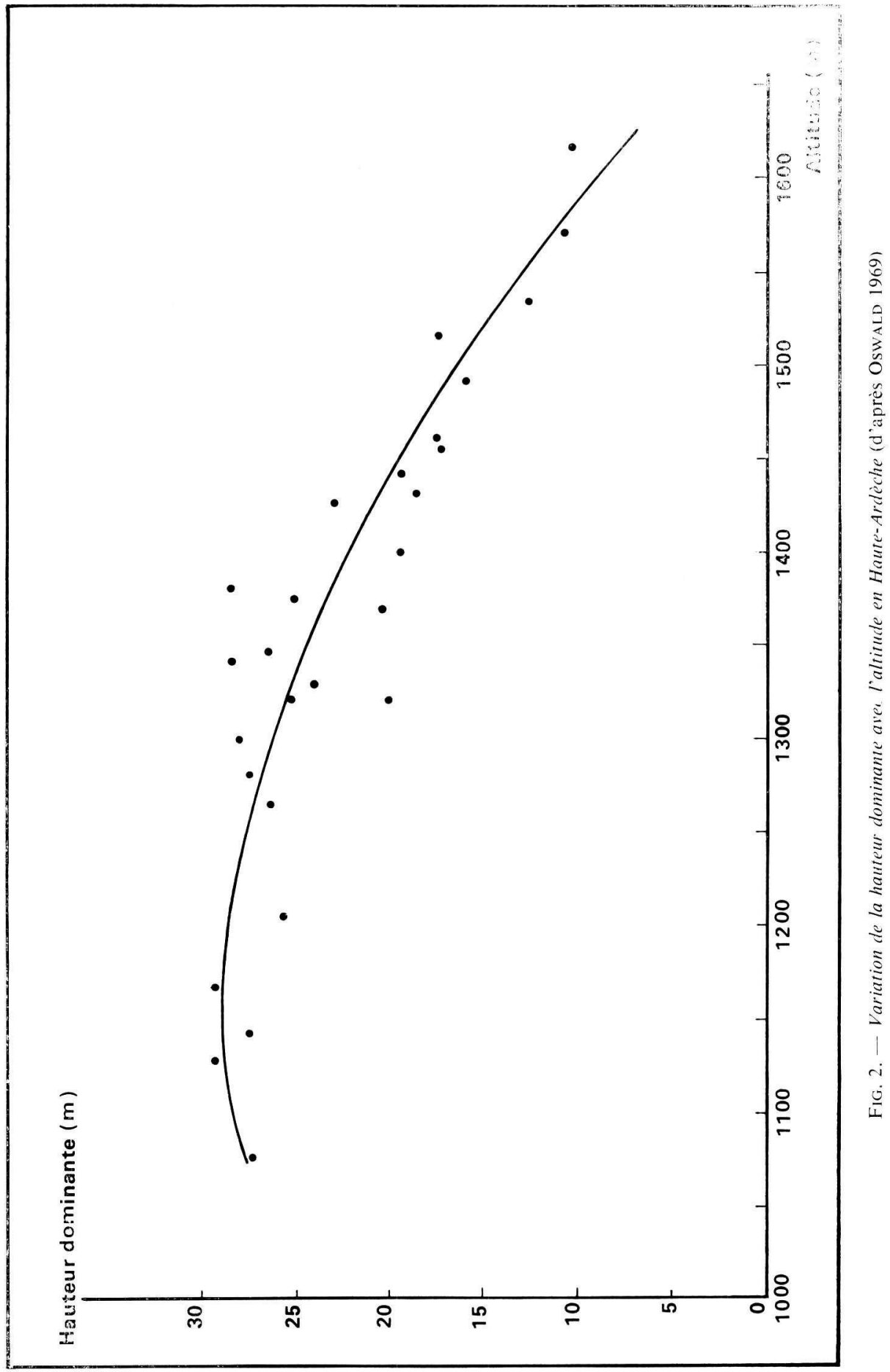


permis de penser que les conditions climatiques des périodes qui ont précédé l'élaboration des bourgeons jouent un rôle déterminant dans l'élongation de l'année suivante. Les études qui ont été faites par différents auteurs : HustisCH (1948), TuRner (1956) montrent qu'effectivement les conditions climatiques et particulièrement la pluviosité de l'année (n-1) jouent un rôle important et quelquefois exclusif pour la pousse de l'année n. Il apparaît cependant que suivant les conditions écologiques (époque du déficit hydrique) de la station et les caractéristiques de croissance de l'espèce (pousse d'août ou non), il faille nuancer cette conclusion. Ainsi dans des recherches que nous avons effectuées en forêt d'Amance à Nancy pour un peuplement d'Abies grandis de 38 ans, il est apparu que, en ce qui concerne le bilan hydrique exprimé par le rapport :

$$
\frac{\mathrm{R}_{(n-1)}+\mathbf{P}_{n}}{\operatorname{ETP}_{n}} \text { avec }
$$

$\mathbf{R}_{(n-1)}$ : réserve en eau à la fin du mois $(n-1)$

$\mathbf{P}_{n} \quad$ : précipitation du mois $n$

$\mathrm{ETP}_{n} \quad$ : évapotranspiration potentielle du mois $n$,

seule l'année $n$ intervenait. L'analyse au niveau individuel avait fait apparaître pour un certain nombre d'arbres, un effet significatif des conditions de l'année $n-1$. Sur la moyenne, cet effet n'est pas significatif, ce qui peut être attribué à des effets génétiques ou à des variations microstationnelles.

Il est probable que les jeunes arbres sont beaucoup plus sensibles aux conditions (particulièrement pour le bilan hydrique) de l'année $n$ que les arbres adultes. Ainsi dans une expérimentation qui consistait à étudier la croissance de jeunes résineux (Epicéa, Sapin, Douglas) en fonction de conditions microclimatiques différentes (lumière, bilan hydrique, etc.), nous avons constaté des différences significatives dès la première année de plantation (fig. $\mathrm{n}^{\circ} 3$ ). Dans une autre expérimentation menée en serre avec Picea abies, planté en pot, la pousse de l'année a été significativement réduite par suite de l'application d'une sècheresse de fin de printemps et de début d'été. Des observations analogues ont été faites par BECKER (1970).

Il est admis à l'heure actuelle que la croissance en diamètre est plus sensible aux conditions de l'année que l'élongation. La multiplication du cambium se produit sur une période plus longue que le développement du méristème apical des bourgeons (fig. 4). Les études effectuées par FritTs (1958) sur le Hêtre ont montré que la croissance en diamètre était expliquée par le bilan hydrique et la température. Au début de la saison de végétation, la température était déterminante puis était remplacée, en été par le bilan hydrique.

L'analyse de la croissance est compliquée par la fructification. L'année suivant une année à forte fructification présente une croissance réduite. La fructification est elle-même sous la dépendance des conditions climatiques. Le cas est particulièrement typique pour le chêne (Quercus sessiliflora, Quercus pedunculata) qui, en France, a des mises à fruit très fréquentes dans l'Ouest et au contraire rares et très inégales dans l'Est. On ne connaît pas exactement les facteurs ou le complexe de facteurs qui expliquent ces différences. L'établissement de corrélations climat-fructifications serait du plus grand intérêt pour le forestier qui désire régénérer ses peuplements. 


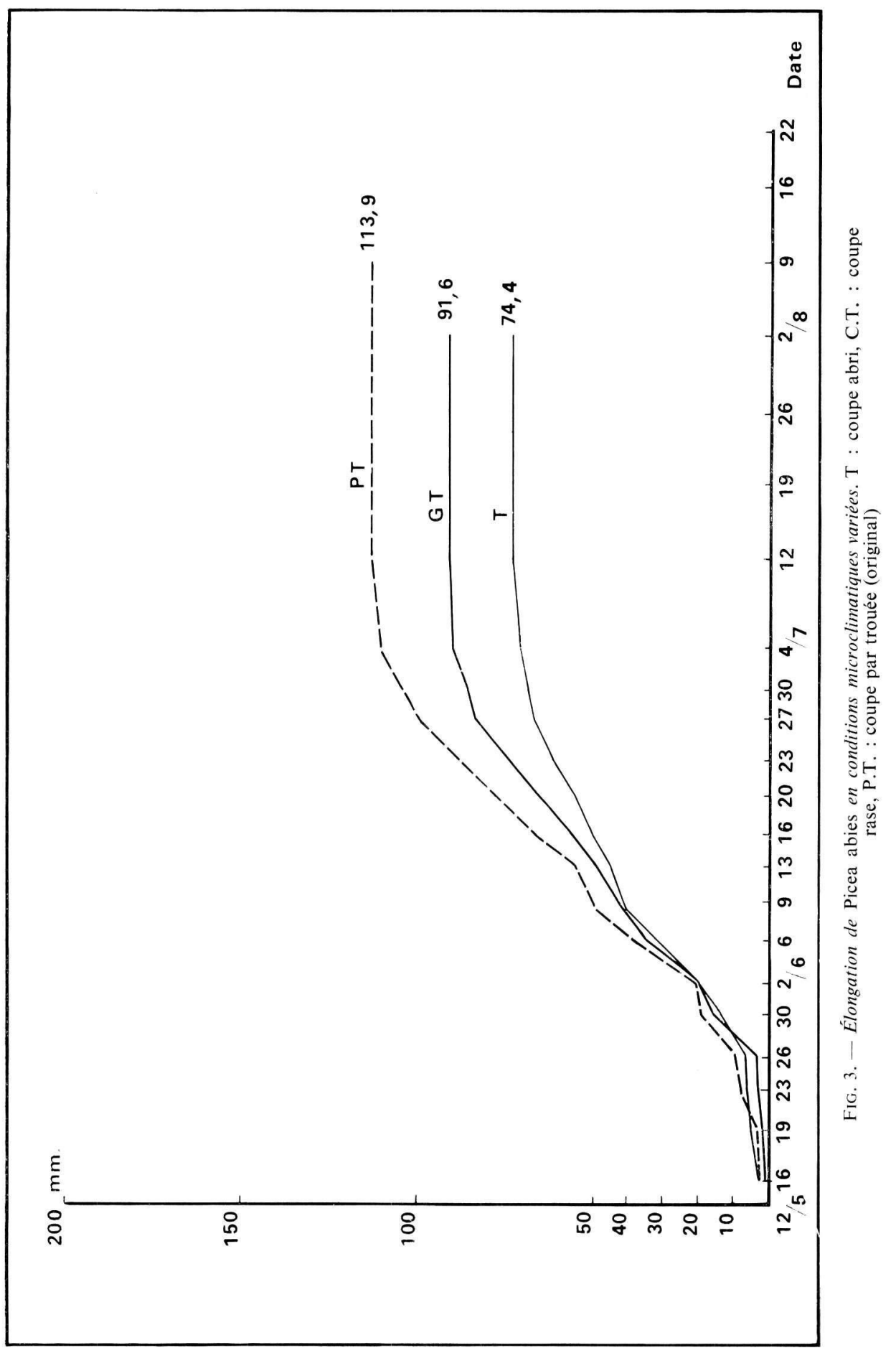




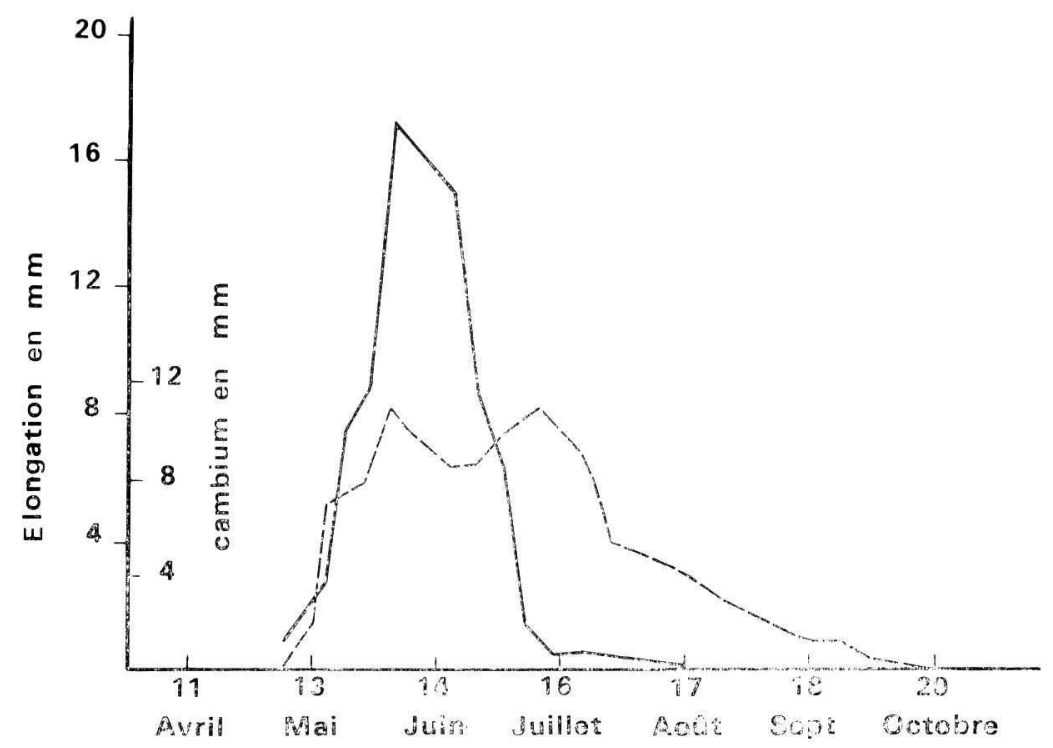

FIG. 4. - Variations saisonnières de la croissance en hauteur et en diamètre chez Pinus strobus (d'après KIENHOLZ 1934)

\section{Études écophysiologiques.}

Les relations entre les facteurs du climat et la production ont été également étudiées par des méthodes écophysiologiques, surtout sur de jeunes arbres, pour des raisons de commodité. Ainsi, des travaux menés en laboratoire par Tranquillini (1955), Kramer (1944), Mitchell (1934), Fairbairn (1970) etc. ont permis de préciser les relations des facteurs climatiques avec les processus photosynthétiques (fig. 5, 6 et 7) dont dépend au premier chef la production ligneuse.

Fairbairn et Neustein ont en particulier montré (fig. $n^{\circ} 8$ ) que, en condition contrôlée, l'élongation passe par un maximum correspondant à une intensité lumineuse inférieure à l'éclairement maximum. Mais le poids de matière sèche est le plus élevé pour l'éclairement maximum, ce qui se traduit par des diamètres plus importants. Ces résultats rejoignent ceux obtenus par Mitchell sur Pinus strobus et par nous-même sur Picea abies. En conditions naturelles, ces effets sont contrebalancés par les autres facteurs (bilan hydrique).

En fait, si ces recherches en laboratoire sont nécessaires, il peut aussi nécessairement travailler en forêt. En effet, d'une part l'action du couvert influence le climat, et d'autre part dans la nature, les facteurs agissent de façon conjuguée et non séparée.

En fait, la compréhension de ces phénomènes passe par deux types de recherches qui sont menées la plupart du temps simultanément : climat

- l'analyse des influences de la structure des peuplements sur les facteurs du micro-

- l'étude de la réponse des arbres à ces facteurs appréciée par la croissance, la production ou la photosynthèse. 


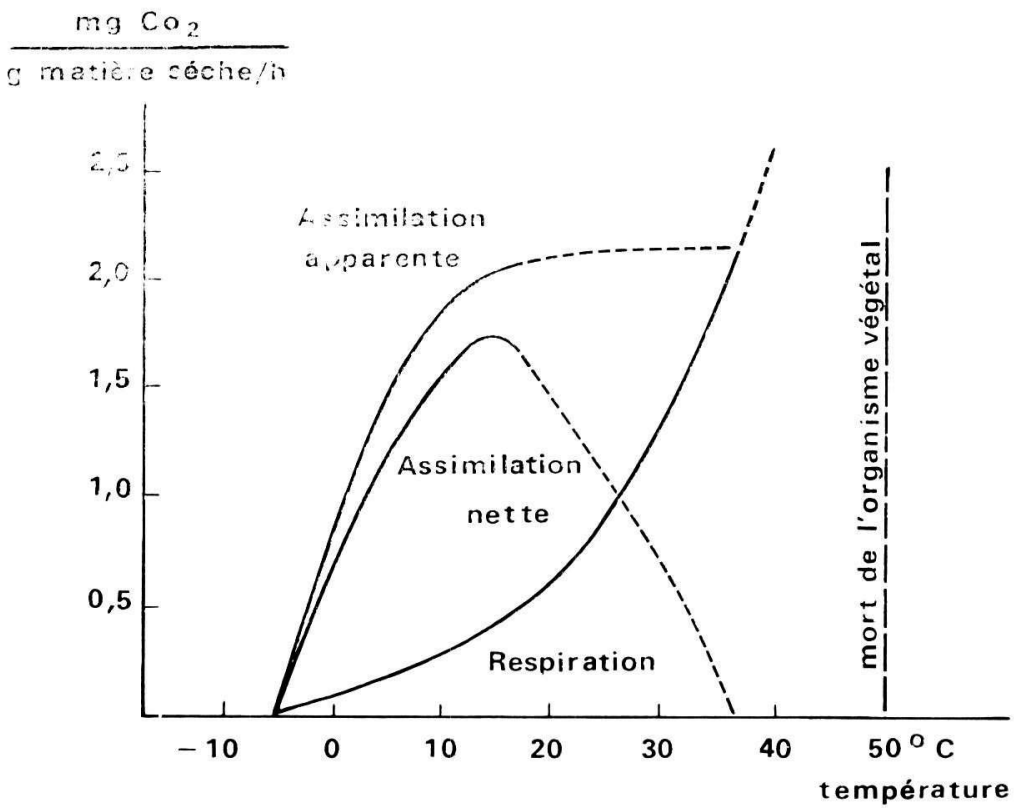

FIG. 5. - Assimilation apparente, respiration et assimilation nette en fonction de la température chez Pinus Cembra (d'après Tranquillini 1955)

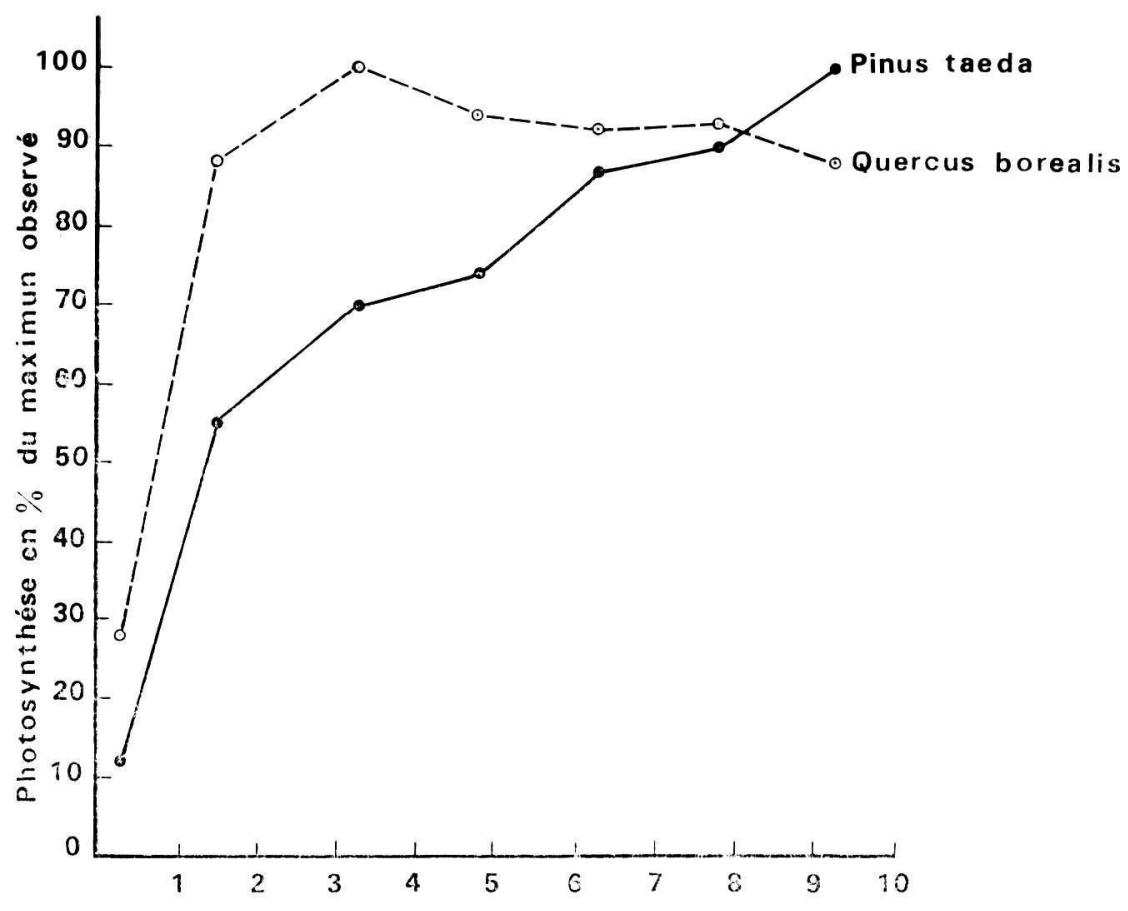

intensité lumineuse en milliers cio foot candles

Fig. 6. - Effet de l'intensité lumineuse sur la photoinghì̀se (d’après Kramer 1944) 


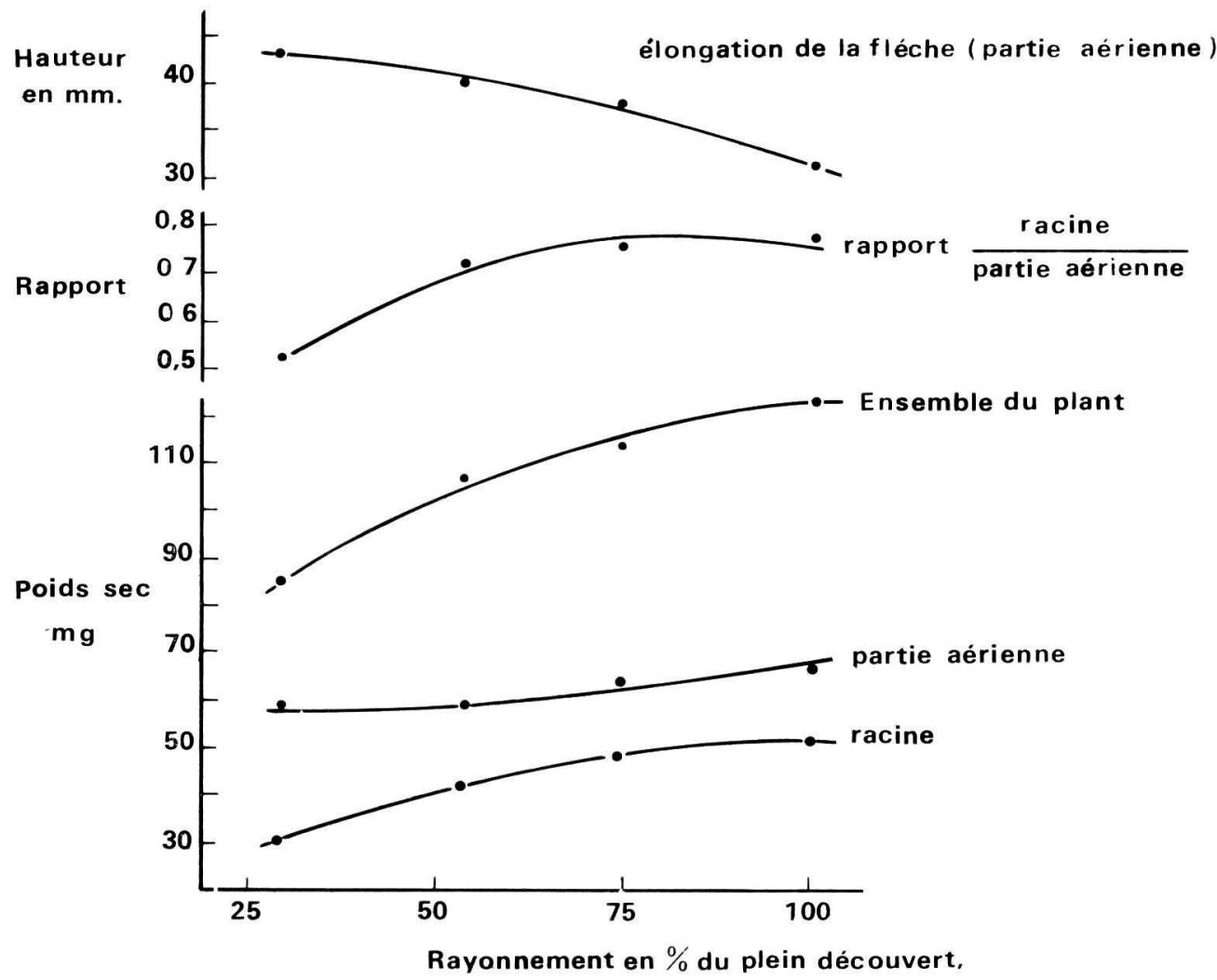

FIG. 7. - Effet de la lumière sur la hauteur de la partie aérienne, le poids sec des racines, le poids sec de la partie aérienne pour des semis de Pinus strobus (d'après Mitchell 1936)

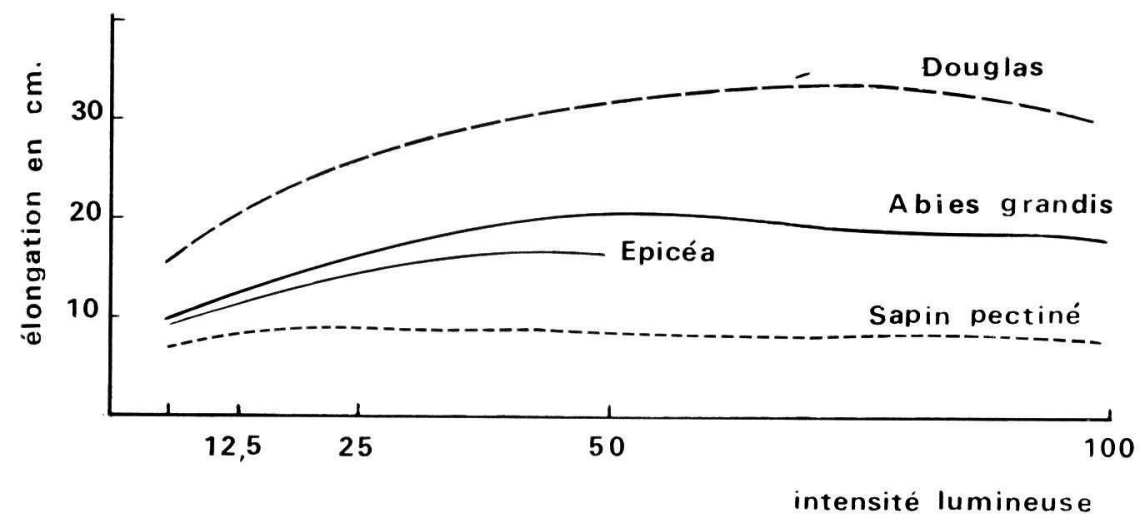

FIG. 8. - Élongation en fonction de l'intensité lumineuse pour des semis de 2 ans (d'après Fairbairn et Neustein 1970) 
Cette démarche présentera à moyen terme l'avantage de connaître, d'une part les influences de tel ou tel traitement sur l'amélioration des conditions de croissance, et d'autre part de prévoir la réponse des arbres avec suffisamment de sécurité. C'est dans ce sens que sont développées les recherches de bioclimatologie forestière à la station de Sylviculture et de Production du C.N.R.F. (Aussenac, Decourt, 1970).

Des études en cours à Nancy devraient nous permettre de préciser pour les régénérations naturelle ou artificielle des résineux, le rôle de la futaie, de la coupe d'abri, de la coupe par bande, par trouée et de la coupe rase.

Bien que l'ensemble des données ne soit pas encore exploitées ni publiées des croissances différentes dans les différents traitements ont pu être expliquées par l'action déterminante de un ou plusieurs facteurs climatiques, Ainsi, dans une comparaison coupe d'abri, coupe par trouée, coupe rase, la meilleure croissance de jeunes résineux pour la coupe par trouée est expliquée par une amélioration du microclimat : diminution du risque de gelées tardives, rapport $\frac{\text { ETR }}{\text { ETP }}$ favorable (AUSSENAC, 1968-1970).

Cette démarche apparaît très fructueuse car elle ouvre des voies de progression pour la recherche des relations facteurs climatiques-production ligneuse.

Ainsi, par exemple, nos travaux ont mis en évidence une morphologie variable de l'appareil foliaire des jeunes résineux introduits dans des microclimats différents. Des recherches sont actuellement en cours pour analyser ces différences tant sur le plan de la morphologie et de l'anatomie des aiguilles que sur celui de la morphologie de la ramification.

De même, les études sur les influences de l'essence et de la structure des peuplements sur la répartition au sol des précipitations nous ont amené, compte-tenu des résultats obtenus, à tester l'importance de ces phénomènes pour la croissance des arbres.

En ce qui concerne plus spécialement les peuplements adultes, les recherches menées par BAUMgartner en Allemagne et la transposition à la forêt des résultats acquis par les chercheurs de la station centrale de Bioclimatologie agricole de Versailles, montrent que l'analyse de l'interaction microclimat-caractéristique du couvert, peut avoir des prolongements pratiques intéressants.

On sait que le rayonnement lumineux est affaibli au fur-et-à-mesure qu'il s'enfonce dans le couvert forestier (loi de Beer). Les conditions d'assimilation vont alors varier suivant que l'on se trouve au sommet ou à la base des houppiers. On sait également que la chlorophylle des différentes essences réagit inégalement à un même éclairement. Les feuilles des essences d'ombre ont, pour un éclairement faible, une photosynthèse plus active que les feuilles des essences de pleine lumière. Un même arbre possède d'ailleurs des feuilles d'ombre et des feuilles de lumière (fig. 9). Par ailleurs Grulors (1967) a montré que pour le Hêtre et le Chêne les angles d'inclinaison des feuilles variaient comme la loi de l'extinction du rayonnement diffus. Le Hêtre présente un tropisme plus marqué et plus sensible que le Chêne.

Il a été possible à BAUMGARTNER (1969) de calculer, niveau par niveau, l'assimilation nette potentielle d'un peuplement d'Epicéa en Bavière (fig. 10). 


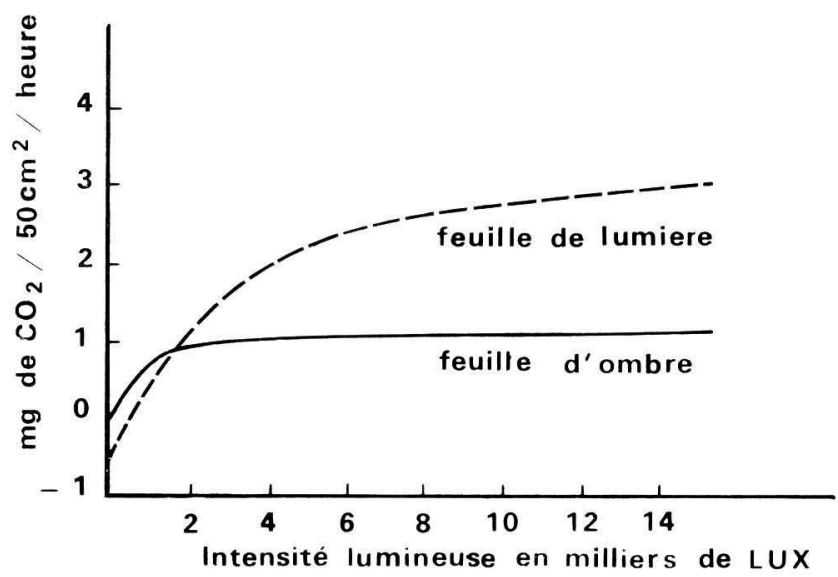

FIG. 9. - Assimilation des feuilles de hêtre : feuilles de lumière et feuilles d'ombre, en fonction de l'intensité lumineuse (d'après BOYSEN JENSEN 1932)
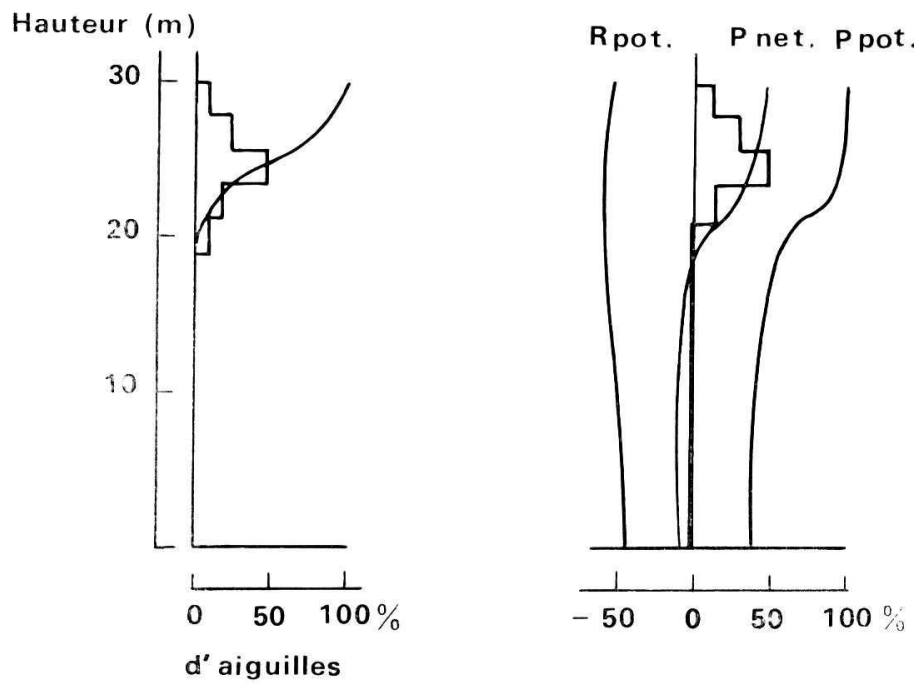

FIG. 10. - Profil vertical de la distribution des aiguilles de la respiration et de l'assimilation dans un peuplement d'Épicea (d'après BAUMgarTNER 1969)

Ces résultats permettent d'entrevoir des applications possibles : étude du rôle de l'élagage artificiel des arbres forestiers sur les processus photosynthétiques, de l'amélioration du potentiel d'assimilation nette par l'utilisation d'essences à morphologie du couvert plus efficace. 


\section{5. - CONCLUSION}

Les relations entre le climat, le microclimat et la production ligneuse sont à la base du développement et de la croissance des peuplements forestiers. Il s'agit donc d'un problème considérable, et l'intérêt des recherches dans ce domaine n'est plus à démontrer. Jusqu'à présent, la pratique forestière a précédé la recherche qui, la plupart du temps, n'a eu qu'un rôle explicatif. Inverser cette succession ne sera possible que dans la mesure où nous serons capables, à partir de recherches de base, de suggérer la façon de modifier au mieux le milieu et les arbres pour aboutir sûrement à la réalisation des objectifs de la sylviculture.

\section{SUMMARY}

\section{CLIMATE, MICROCLIMATE AND WOOD PRODUCTION}

The combined action of climatic parameters and especially of thermal and hydronietric factors rules the life conditions of trees and determines the geographic distribution of the differents species and types of forests.

Researches in forestry should allow a better control of the forest by stating the forest potential of various sites, the climatic requirements of the main tree species and the means to improve the relations between tree and microclimate.

Climatic or microclimatic factors may act in two ways :

- accidentally (climatic accidents),

- permanently (physiological action).

Relations between climate and wood production have been studied from production indices such as PATERSON's index which is also useful to the economist for a rough estimate of the production of a country. But the estimate of the potential wood production is difficult, because, on the one hand, forests lie mostly uncultivated soils (the least fertile) and, on the other hand, introduction of exotic species may increase this production.

On a regional scale, studies of forest potentialities may induce interesting information, but are limited for want of climatological data. Therefore it will be difficult to reach a thorough knowledge in this fiels.

At the level of a forest or a plot, the canopy influences the climatic factors and we speak of microclimate. The studies « microclimate-production » have to take these influences into account. Special notions occur : the structure of crowns and stands, the environment of the experimental plot.

Numerous studies have been done under controlled conditions and have allowed to specify the relations between photosynthetic processes and climatic factors. But it is necessary to work within the forest. On the one hand the canopy influences the climate and on the other, factors do not act separately in nature, but jointly. These phenomena can be cleared through two kinds of research which are often led simultaneously as ours in Nancy :

- the analysis of the influences of stand structures of microclimatic factors, synthesis.

- the study of the response of trees to these factors in terms of growth, production or photo-

Likewise the study of the role of forest canopy allows to consider some pratical measures : influence of artificial pruning, improvement of the net assimilation capacity by choosing species with a more efficient morphology of the crown.

\section{RÉFÉRENCES BIBLIOGRAPHIQUES}

AussenaC G., 1968. - Observations à propos d'une gelée tardive, R.F.F., (3), 204-211.

Aussenac G., 1970. - Gelées tardives et jeunes peuplements forestiers, R.F.F., XXII (4), 463-469. 
Aussenac G., 1970. - Action du couvert sur la distribution au sol des précipitations. Ann. Sci. forest., 27, (4), 329-389.

Aussenac G., Decourt N., 1970. - Écophysiologie, Sylviculture et Aménagement. Exposé IUFRO, section 23, $11 \mathrm{p}$.

Baumgartner A., 1969. - Meteorological approach to the Exchange of $\mathrm{CO}_{2}$ between the Atmosphere and Vegetation, particularly Forest stands. Photosynthetica, 3 (2) 127-149.

BeCKer M., 1970. - Transpiration et comportement vis-à-vis de la sécheresse de jeunes plants forestiers (Abies alba, Picea abies, Pinus nigra et Pinus strobus). Ann. Sci. forest., 27 (4) 401-420.

Boysen-Jensen P., 1932. - Die Stoffproduktion der Pfanzen - Gustav Fischer Verlags BuchlandungGermany.

Chartier P., 1966. - Étude du microclimat lumineux dans la végétation. Ann. Agron, 17, (5) 571-602.

Ducrey M., 1971. - Contribution des photographies hémisphériques à l'étude du microclimat lumineux. Pub. Station Sylviculture et Production, $19 \mathrm{p}$.

Fairbairn W. A., Neustein S. A., 1970. - Study of response of certain coniferous species to light intensity. Forestry, 43, (1) 57-71.

FreEland R. O., 1952. - Effect of age of leaves upon the rate of photosynthesis in some conifer. Plant Physiology, 27, 685-690.

Fritrs M. C., 1958. - An analysis of radial growth of beech in a central Ohio forest during 1954-1955, Ecology, 39, 705-720.

Grulois J., 1967. - Extinction du rayonnement global, Tropismes et paramètres foliaires. Bull. Soc. Roy. Bot. Belgique, 100, 315-333.

Hustich I., 1948. - The Scotch pine in northemmost Finland and its dependance on the climate in the last decades, Acta Bot. Fennica, 42, 4-75.

Keinholz R., 1934. - Leader, needle, cambial and root growth of certain conifer and their relationship. Bot. gaz., 96, 73-92.

Kramer P. S., Decker J. P., 1944. - Relation between light intensity and rate of photosynthesis of loblolly pine and certain hardwoods. Plant-Physiology, 19, 350-358.

Mitchell H. L., 1934. - Pot culture tests of forest soil fertility. Black Rock. Forest Bull., 5.

Oswald H., 1969. - Conditions forestières et potentialité de l'épicéa en Haute-Ardèche. Ann. Sci. forest., 26, (2) 183-224.

Parde J., 1959. - Une notion nouvelle et fructueuse : l'indice C.V.P. R.F.F., 195-200.

PARde J., 1963. - Retour sur l'indice C.V.P. de Paterson. R.F.F. 1.

PATERSON S. S., 1956. - The forest area of the world and its potential productivity. Göteborg, 216.

Perrin H., 1963. - Bases scientifiques de la sylviculture, École Nationale des Eaux et Forêts, 1-303.

Tranoulllini W., 1955. - Die Bedeutung des Lichtes und der Temperature für die Kohlensäme-assimilation von Pinus cambra Jungwachs an einem hochalpinen Standort. Planta, 46, 156-178.

TURner R. M., 1956. - A study of some features of growth ans reproduction of Pinus ponderosa in northern Idaho. Ecology, 37, 742-753.

Van Miegreet, 1967. - La définition du but de la sylviculture. Sylva gandensis, (1) 1-20.

WECK J., 1960. - Die Waldformations Klassen der Erde und ihre potentielle Substanzerzengung Sonderabdruck aus Scientia 54, 6, Série S.1-3.

\section{DISCUSSION ET INTERVENTIONS}

M. Pourtet. - Monsieur Aussenac a dit que le bilan hydrique de l'année influait plus sur l'accroissement en diamètre que sur l'accroissement en hauteur.

Il me paraît qu'il faut distinguer Conifères et Feuillus.

1) Conifères. L'accroissement en hauteur se produit en quelques semaines au printemps; il paraît surtout être influencé par le bilan hydrique de l'année précédente.

Dans les plantations de Pins Noirs des calcaires jurassiques du Nord-Est de la France, l'été sec 1949 s'est traduit dans tous les peuplements par une très faible pousse en hauteur 1950.

2) Feuillus. C'est probablement moins net pour la hauteur en ra ison du plus grand étalement de l'accroissement. 
Pour le Peuplier, c'est en quelque sorte le «climat courant » qui influe immédiatement et directement sur l'accroissement courant en diamètre.

Pour de jeunes plants de Peuplier à Verreuil, près Blois, dont la circonférence était mesurée chaque semaine, l'accroissement hebdomadaire variait de $1 \mathrm{~mm}$ à $10 \mathrm{~mm}$ sur la circonférence, le plus faible se produisait pendant les semaines froides et sèches, le plus fort pendant les semaines humides et chaudes.

M. Aussenac. - Pour les résineux, nous avons observé à Nancy que l'accroissement hebdomadaire en longueur variait avec la température moyenne de la semaine, et dans le même sens.

M. Catinot. - La formule de Paterson appliquée aux forêts tropicales donne des résultats très optimistes : 12 à $15 \mathrm{~m}^{3} /$ ha/an contre 2 à $3 \mathrm{~m}^{3} /$ ha/an réels.

Ne serait-ce pas dû au fait que la formule de PATERson cerne mal l'influence de la température sous climat tropical, car on sait maintenant (MüLLER et J. NIELSEN) que plus de $75 \%$ de la production brute (chlorophyllienne) sont consommés par la respiration sous le climat de la Basse Côte d'Ivoire où la température moyenne se situe autour de $25^{\circ}$.

M. Aussenac. - Entièrement d'accord.

M. Guillard. - Réponse à M. Catinot.

1) Les données de JÖRGENSEN sur la productivité primaire de la forêt tropicale ont bien montré la faiblesse de la production ligneuse due à l'abondante consommation due à la respiration.

2) Holdridge dans son concept de Life zone ne considère que les «biotempératures » $\left(0-25^{\circ}\right)$, ce qui permettrait de tempérer singulièrement l'indice C.V.P. lui-même très grossier - et donner des résultats plus réalistes pour les forêts tropicales.

M. LemÉE. - J'ai beaucoup apprécié le souci de M. Aussenac de relier les études écophysiologiques expérimentales aux observations sur les actions climatiques sur la production. C'est ainsi que la productivité nette relativement faible de la forêt tropicale pluvieuse, évoquée par M. CATinOT, est manifestement la résultante d'un bilan « fixation photosynthétique brute du $\mathrm{CO}_{2}$ /consommations respiratoires » favorable au second terme en raison des températures nocturnes élevées.

M. Vernet. - L'étude des profils hydriques sous forêts donnent des renseignements précieux sur le transfert des réserves d'eau accumulées en hiver vers les saisons à forte E.T.P.

Par exemple, à Versailles, l'excédent d'hiver d'environ $200 \mathrm{~mm}$ permet de combler le déficit estival qui est de l'ordre de $200 \mathrm{~mm}$, à condition que le sol soit assez profond (1,50 $\mathrm{m}$ à $2 \mathrm{~m})$.

En Sologne, à la fin d'un été sec, nous avons constaté que sous chênes, des limons étaient desséchés jusqu'à près de $2 \mathrm{~m}$ de profondeur. Sous couvert faible de Bouleau, les sables étaient humides à faible profondeur. Suivant les sols, la compensation peut être très différente et, par suite, la croissance très variable.

M. Aussenac. - Il semble que sous le climat lorrain, l'évapotranspiration réelle de peuplements adultes et fermés soit sensiblement la même pour différentes essences. 
M. LÉVY. - Les conditions climatiques interviennent d'une façon différente suivant les caractéristiques du sol. Mais c'est un problème très complexe, car le sol intervient sur l'alimentation en eau des arbres, non seulement par sa capacité de rétention en eau utile, mais également par son action sur la profondeur d'enracinement.

M. Bonneau. - La réserve en eau utile du sol a bien sûr une importance : voir par exemple l'étude de LE TACON sur l'épicéa dans le Nord-Est, où la production dépend de la réserve en eau utile.

De plus, cette notion de réserve en eau utile est très difficile à saisir : la profondeur du sol est une notion très floue. La mobilité des réserves intervient : par exemple les réserves en sol sableux paraissent utilisables dans leur presque totalité (opinion confirmée par Bouchet, qui dit que les sables sont d'excellents " pièges à eau »).

M. Defontaines. - Oswald a montré que l'optimum de productivité de l'épicéa dans la Haute-Ardèche se situait autour de $1000 \mathrm{~m}$. A-t-on une idée des altitudes auxquelles correspond cet optimum dans les différents massifs montagneux en France.

M. Oswald. - L'optimum de production d'un massif montagneux dépend essentiellement de l'altitude de la limite potentielle supérieure de la forêt.

La diminution de la production devient de plus en plus rapide dès qu'on s'approche de la limite supérieure.

M. Bourgenot. - On ne connaît pas de résultats précis, sauf dans quelques cas limités, lorsque l'on dispose d'inventaires de forêts.

M. Pourtet. - L'effet de l'altitude n'est pas seul en jeu pour limiter la végétation ligneuse et la production.

L'effet de «sommet » et du vent est capital : ex. à $300 \mathrm{~m}$, au Pays de Galles, cet effet empêche toute plantation ligneuse.

M. BARNÉOUD. - Des expériences de fertilisation forestière semblables, sur des sols semblables, donnent des résultats très variables, suivant la région. Le climat semble le facteur principal : y a-t-il eu des études pour déterminer à quel niveau tel facteur climatique limite l'action de l'engrais?

M. Bonneau. - En altitude, les effets de la fertilisation restent positifs, mais très réduits en valeur absolue à cause du facteur limitant qu'est l'altitude.

M. Bourgenot. - Pour comparer les productions entre espèces en fonction de l'altitude, il faut également tenir compte de la qualité (dégâts dûs à fomes annosus sur l'Épicéa en basse altitude).

M. LANiER. - L'optimum de croissance pour l'Épicéa en Haute-Ardèche a été trouvé vers $1000-1100 \mathrm{~m}$. On a indiqué que pour le Sapin, l'optimum de croissance a été trouvé vers $800 \mathrm{~m}$ dans le Jura et $600 \mathrm{~m}$ dans les Vosges.

Peut-on savoir si l'optimum ainsi défini est indépendant de l'essence ou non, c'est-àdire par exemple si l'optimum de croissance en Ardèche se situerait également pour le Hêtre ou le Sapin à la même altitude de $1000-1100 \mathrm{~m}$ ?

Personnellement, je pense que non, et que cet optimum est fonction directe de l'essence. 
M. Bouvarel. - Dans une situation de forêt naturelle, avec une succession d'espèces, au fur et à mesure qu'on s'élève en altitude, a-t-on une courbe globale avec un optimum ou une suite de courbes spécifiques?

M. Sauvage. - Comme suite aux interventions de Lanier et de Bouvarel, il serait donc très intéressant d'étudier l'optimum de croissance pour l'Épicéa qui intervient, d'une part dans les forêts du $2^{\mathrm{e}}$ plateau, mais avec le Hêtre et le Sapin, d'autre part dans le Jura plissé.

M. Тотн. - La production optimale pour le Pin Noir d'Autriche est de $8-10 \mathrm{~m}^{3}$ / ha/an à 1 000-1 $250 \mathrm{~m}$ d'altitude en France (Alpes, Mont Ventoux).

Il y a une différence entre exposition Sud et Nord.

3 facteurs importants :

1) climat,

2) altitude - exposition,

3) type de sol.

M. BALDY. - Il y a des variations importantes de productivité nette de graminées pérennes en fonction de leur origine géographique (et génétique). On a des différences environ $10^{\circ} \mathrm{C}$ d'optimum de la photosynthèse nette de la Suède au Portugal pour des variétés originaires de ces zones. Ceci est lié à la modification de l'optimum de la photorespiration. Des études analogues sont conduites sur des acacias.

L'origine génétique et l'optimum thermique sont étroitement liés. Il faut s'en préoccuper dans les introductions.

M. Bouvarel. - Les expériences de Clausen sur les potentilles, en Californie, ont permis de mieux connaître les mécanismes de l'adaptation héréditaire des écotypes aux conditions du milieu, et la diversité, dans une même espèce, des réponses à des conditions de milieu différentes de celles du lieu d'origine. Certains écotypes ont des exigences strictes, et perdent leur vigueur lorsqu'ils sont déplacés, d'autres sont beaucoup plus " plastiques ». Les croisements entre écotypes donnent naissance à des populations hybrides dont certaines manifestent une vigueur supérieure aux populations autochtones dans des conditions de milieu différentes.

M. Defontaines. - Pour aborder des problèmes d'Aménagement du Territoire, il semble qu'il soit utile de travailler au $1 / 200000^{\mathrm{e}}$. Peut-on utiliser l'indice C.V.P. à cette échelle?

M. Aussenac. - Au 1/200 000e, dans un climat tempéré frais, l'indice C.V.P. risque d'être imprécis. S'il s'agit d'une carte recouvrant des régions à climats contrastés, cet indice pourra être alors d'une grande utilité.

M. Decourt. - Le C.V.P. ne peut servir que de garde fou à des aménagistes du territoire dans le domaine de la production forestière.

Les spécialistes forestiers n'en ont pas l'usage à l'échelle de la région.

M. Fougerouze. - La rareté des observations climatologiques valables, à diverses échelles de temps et d'espace, tient à des causes diverses :

- technologie d'acquisition datant du siècle dernier et nécessitant une intervention humaine quotidienne; fiabilité faible; 
— rareté de techniques d'approche à l'usage des écologistes, permettant la semiautomatisation de la collecte des données à un prix abordable;

- grande diversité des activités dans le secteur climatologique, non coordonnées entre elles et vouées à une audience limitée, du fait de l'incertitude des relevés et de l'inexistence d'une normalisation.

Ces hypothèques peuvent être levées en organisant la coordination à l'échelle nationale, en poussant les études technologiques et les organisations de réseaux.

M. Bouvarel. - Entre les données climatiques et les données microclimatiques, il y a un fossé : celui des données mésoclimatiques, qui manquent cruellement aussi bien au forestier gestionnaire qu'à l'écologiste. Il faudrait multiplier les réseaux d'observations mésoclimatiques, du type de celui qui a été installé au Ventoux en liaison avec les entomologistes.

M. Gounot. - Les besoins de l'aménagement du territoire nécessitent des vues synthétiques englobant le climat, le sol et la végétation à une échelle de l'ordre du $200000^{\mathrm{e}}$. Un tel travail a été effectué en Tunisie et fournit tous les renseignements indispensables de ce point de vue. Il fournit également un cadre précis pour la définition des groupes écologiqus.

M. BonNEAU. - L'étude d'un cadre régional sur le plan scientifique est certainement indispensable, mais cette base peut ne pas être cartographique. Une carte au 1/200 000 peut n'apprendre au gestionnaire que des choses qu'il connaît déjà de manière intuitive ou expérimentale.

M. Grandjean. - Il est nécessaire d'étudier les groupements écologiques à petites échelles avant de passer à une cartographie détaillée du massif forestier à l'échelle du $1 / 5000^{e}$ — voire du $1 / 10000^{\mathrm{e}}$. 